\title{
Molecular Target Discovery Grants
}

National Cancer Institute

\section{Source}

National Cancer Institute. Molecular Target Discovery Grants. NCI Thesaurus. Code C19968.

Proposed mechanism for providing resources for "mechanisms" researchers to provide evidence that a proposed new cancer relevant molecule is a good target for drug discovery. (Bypass Budget) 